(C) 2018 IEEE. Personal use of this material is permitted. Permission from IEEE must be obtained for all other uses, in any current or future media, including reprinting/republishing this material for advertising or promotional purposes, creating new collective works, for resale or redistribution to servers or lists, or reuse of any copyrighted component of this work in other works. 


\title{
Calibration of a Rotating Laser Range Finder using Intensity Features
}

\author{
Kavindie Katuwandeniya ${ }^{1 \dagger}$, Ravindra Ranasinghe ${ }^{1 \ddagger}$, Lakshitha Dantanarayana ${ }^{1}$, Gamini Dissanayake ${ }^{1}$, \\ and Dikai Liu ${ }^{1}$
}

\begin{abstract}
This paper presents an algorithm for calibrating a "3D range sensor" constructed using a two-dimensional laser range finder (LRF), that is rotated about an axis using a motor to obtain a three-dimensional point cloud. The sensor assembly is modelled as a two degree of freedom open kinematic chain, with one joint corresponding to the axis of the internal mirror in the LRF and the other joint set along the axis of the motor used to rotate the body of the LRF. In the application described in this paper, the sensor unit is mounted on a robot arm used for infrastructure inspection. The objective of the calibration process is to obtain the coordinate transform required to compute the locations of the $3 \mathrm{D}$ points with respect to the robot coordinate frame. Proposed strategy uses observations of a set of markers arbitrarily placed in the environment. Distances between these markers are measured and a metric multidimensional scaling is used to obtain the coordinates of the markers with respect to a local coordinate frame. Intensity associated with each beam point of a laser scan is used to locate the reflective markers in the $3 D$ point cloud and a least squares problem is formulated to compute the relationship between the robot coordinate frame, $L R F$ coordinate frame and the marker coordinate frame. Results from experiments using the robot, LRF combination to map a cavity inside a steel bridge structure are presented to demonstrate the effectiveness of the calibration process.
\end{abstract}

\section{INTRODUCTION}

Three dimensional range sensors that can provide direct geometric information of the environment are vital in many robot applications. When accurate geometric information is required at a fast rate, particularly from a moving platform, the method of choice is a 3D laser range finder such as the Velodyne [1]. The low cost, low accuracy, short range alternative is a depth camera such as Intel RealSense [2]. Literature is abound [3], [4], [5] on how such sensors can be calibrated so that the acquired 3D information can be registered with respect to a coordinate frame attached to the robot on which the sensor unit is mounted. In applications where a 3D point cloud is needed to be acquired while stationary; a high quality 2D scanning laser range finder (LRF) such as the Hokuyo UTM-30LX-EW, can be used to build a 3D range sensor by rotating the LRF body using a servo motor resulting in low cost, high accuracy and long range alternative. The direction of the laser beam at any given instant is a function of both the servo motor rotation and the scanning system within the LRF. The acquired data from the Hokuyo LRF corresponds to the distance to the nearest

1 Centre for Autonomous Systems (CAS), University of Technology Sydney, Australia

$\dagger$ This work was conducted during an internship at CAS, University of Technology Sydney, Australia.

$\ddagger$ Corresponding Author: ravindra.ranasinghe@uts.edu.au occupied region of the environment from the optical centre of the LRF.

To capture the geometry of the environment, it is necessary to describe the locations of these points with respect to a fixed coordinate frame. Nominal values of extrinsic parameters of a laser range finder constructed as outlined above, which are available in the design drawings, can be used for this purpose. The focus of this paper is to formulate a calibration strategy to refine these nominal values to take into account the changes that occur during manufacture and assembly.

The paper presents a solution to find the Homogeneous transformation matrix (HTM) relating the coordinate frame of the robot and the 3D range sensor constructed as described above. Geometry of the range sensor is described using four Denavit-Hartenberg (DH) parameters that define the relationship between the two rotary axes of the range sensor. A set of reflective markers are arbitrarily placed in the environment and the distances between these markers are measured. Multidimensional scaling is used to obtain the coordinates of the markers with respect to a local coordinate frame. Intensity value associated with each beam point acquired through the LRF is used to identify the reflective markers in the point cloud and thereby measure ranges to the markers. A non-linear least squares optimization problem is formulated and solved to obtain the calibration parameters required to transform the laser observations into the robot coordinate frame. Although intensity images of checkerboard calibration patterns have been used to help calibrate laser range finders [6], [7] to the best of our knowledge, this is the first time return laser intensity is used in this fashion in a laser calibration process.

The work presented in this paper was motivated by a collaborative project that aims to develop a robot for the cleaning and painting inside the arch structures of the Sydney Harbour Bridge (SHB). The arches of this bridge is constructed by joining rectangular shape hollow steel sections (box girders) with rivets to hold the structure in place. Changes in the New South Wales (NSW), Australian government's Work Health and Safety (WHS) legislation [8] have resulted in some areas outside the bridge and all the confined areas inside the hollow sections being inaccessible to workers making a robotic solution essential. The robot will navigate inside these box girders of the arch structures in the SHB to locate the areas of interest to clean and paint. Once the robot is deployed via an access way at the top of a tunnel section, it is expected to move through several sections which are separated by steel plates on which 
there is a hole sufficient enough for a human to crawl through. Hence, identifying the $6 \mathrm{DoF}$ pose of the robot base (i.e. robot localization) relative to the tunnel section is a prime requirement to accomplish the above. Moreover, identification of the relative $6 \mathrm{DoF}$ pose of end-effectors such as cleaning and painting tools and the location of areas of interest to be cleaned and painted relative to the robot are of greater importance to perform the expected tasks accurately. Therefore, availability of a detailed 3D map of the tunnel section is an indispensable requirement to understand the surface geometry which is important for planning the robot motion.

This paper is organized as follows. Sec. II presents a brief review of related work in extrinsic calibration of $2 \mathrm{D}$ LRF module. Sec. III describes the procedure carried out in the calibration process based on the proposed optimization method. Experiments that were performed to validate the proposed algorithm are presented in Sec. IV. Sec. V reports calibration results from both simulation and real world experiments. Sec. VI concludes the paper.

\section{RELATED WORK}

The extrinsic calibration of the 2D scanner with an extra rotation has been studied during the past years. The intrinsic calibration method proposed in [9] obtains orientation parameters using a plane-based technique. A single scan of a plane is used to iteratively optimise the calibration angles by maximizing the flatness and the inliers of the scanned plane. Kim et al. [10] have proposed a method that requires an exhaustive search to find the extrinsic parameters between the motor and the LRF. They verified the accuracy of the technique by evaluating the surface normal of the calibration structure which was adjusted to be in the same direction as the $x$ axis of the motor coordinate system. A technique to calibrate scans from a LRF to a vehicle-centred reference frame was conducted by acquiring two sets of corresponding three-dimensional points and finding the rigid transformation that maps one onto the other with least squared error [11]. The target of calibration was attached to the leg of the robot and a transformation was found between the scanner and the rigid body.

Many research activities have been conducted in the area of finding the transformation between multi sensor systems mostly consisting of a camera and a LRF using a calibration pattern [12], [13], [14]. Laser-Camera Calibration Toolbox (LCCT), a Matlab ${ }^{\circledR}$ based graphical user interface were used in the calibration procedure presented in [15]. To find the axis of rotation relative to the mirror centre of the LRF, a method is proposed in [16] uses planar checkerboard patternswith the aim of finding the four parameters corresponding to the rotational motor axis and the extrinsic calibration between the rotational axis and a camera. In [7], an extendable framework is proposed to calibrate the robot's joint offsets and external sensor locations based on combining the measurements of various sensors of the robot. The proposed calibration method uses the bundle adjustment

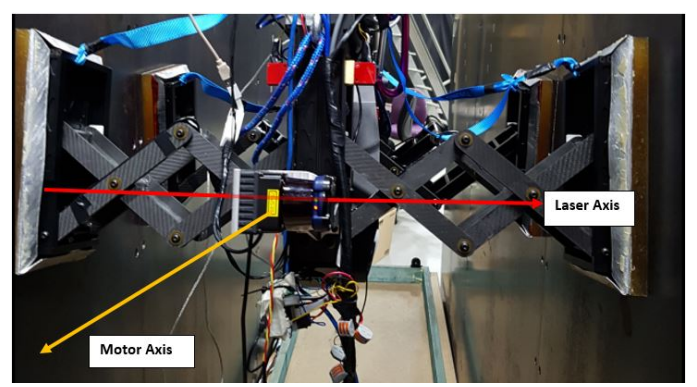

Fig. 1: Kinematic structure of the 3D laser sensor

framework and is implemented on the PR2 ${ }^{\mathrm{TM}}$ mobile manipulation platform. The method proposed in [6] calibrates a twoaxis scanning lidar using a checkerboard calibration target where the lidar is generalized as an idealized special camera with additive measurement distortion. Proposed algorithms in both [7] [6] papers, an intensity image is used in conjunction with a checkerboard pattern. Methodology proposed in [17] finds the rotation axis and the rotation radius by solving a linear equation constructed from a point-plane constraint established by scanning a checker-board calibration pattern in several poses. Using the decoupling property of which a line on a plane does not contain $3 \mathrm{DoF}$ translation terms, a method was proposed in [18] to find the six parameters between the two centres of LRF and a rotating platform. Further, to increase the accuracy of constructed point clouds, it was suggested to perform internal calibration [19], [20], [21]. These methods are not applicable in the work presented in this paper as the robot that we develop to deploy in SHB does not use a camera and a laser together in constructing the $3 \mathrm{D}$ point clouds.

\section{Methodology}

\section{A. Defining the Coordinate Systems}

The aim of the paper is to find the homogeneous transformation matrix that defines the relationship between the coordinate system set up on the motor axis and the optical centre of the LRF. The relationship between the above two coordinate frames can be completely defined using $\mathrm{DH}$ parametrisation with four unknown parameters.

The links associated with the definition are the motor axis and the laser axis as depicted in Fig. 1. For generality, links are assumed to be non-intersecting with a common normal as shown in Fig. 2. Now, the coordinate frames as illustrated in Fig. 3 and Fig. 4 can be established.

1) W - World Coordinate System

2) M - Fixed coordinate frame attached at the point of intersection between the motor axis and the common normal. Its $z$ axis is along the motor axis and the $x$ axis along the common normal.

3) $\mathbf{M}^{\prime}$ - Coordinate frame attached at the point of intersection between the motor axis and the common normal which rotates with the motor. The $z$ axis is along the motor axis and the $x$ axis along the common normal. When motor encoder reading is zero, $\mathbf{M}^{\prime}$ coincides with $\mathrm{M}$. 


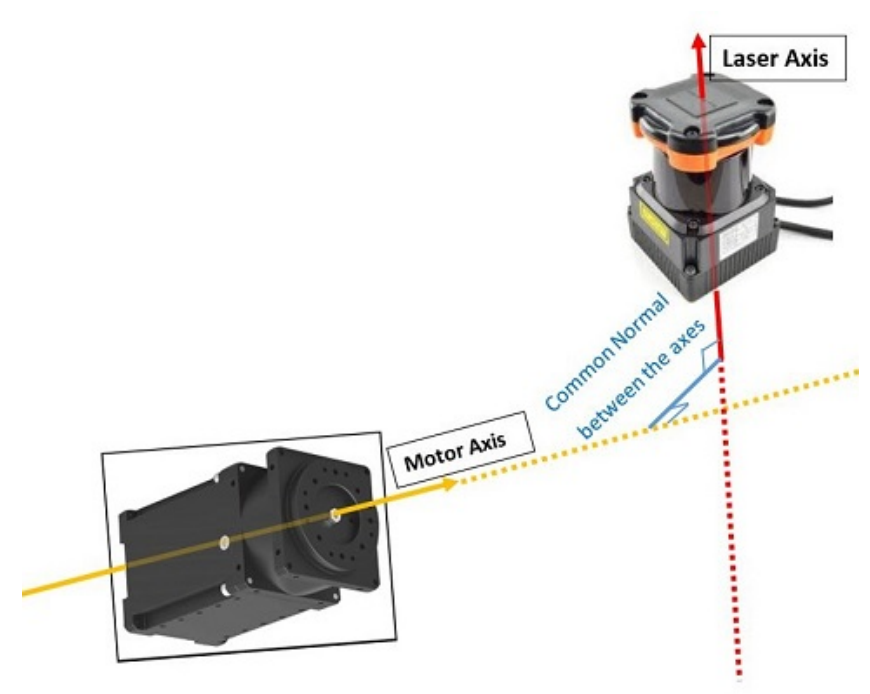

Fig. 2: Common Normal between the links

4) I - Coordinate frame attached at the point of intersection between the laser axis and the common normal by moving $\mathrm{M}^{\prime}$ along its $x$ axis (i.e. the common normal)

5) $\mathrm{I}^{\prime}$ - Coordinate frame attached at the point of intersection between the laser axis and the common normal by rotating I until its $z$ axis coincides with the laser axis. This has its $z$ axis along the laser axis and the $x$ axis along the common normal.

6) $\mathrm{L}^{*}$ - Coordinate frame attached at the optical centre by moving $\mathrm{I}^{\prime}$ along its $z$ axis (i.e. the laser axis)

7) $L^{\prime}$ - Coordinate frame attached at the optical centre by rotating $\mathrm{L}^{*}$ around its $z$ axis until its $x$ axis coincides with the $0^{\text {th }}$ beam encoder position of the laser

8) L - Coordinate frame attached at the optical centre which rotates with the laser beam. This coordinate frame has the same origin and $z$ axis as $\mathrm{L}^{\prime}$. When the beam encoder reading is zero, $\mathrm{L}^{\prime}$ coincides with $\mathrm{L}$.

The world coordinate frame $\mathrm{W}$ is arbitrarily set as described in Sec. III-C. Frame $\mathbf{M}^{\prime}$ moves with the rotation of the motor while frame L moves with the laser beam. All the other coordinate frames are static frames fixed with respect to frame $\mathrm{W}$.

\section{B. Variables of interest}

According to the coordinate frames established, following 10 variables are to be determined.

- 6 variables from $\mathrm{W}$ to $\mathrm{M} ; 3$ for translation $\left(x_{w}, y_{w}, z_{w}\right)$ and 3 for rotation ( $\psi_{w}($ roll $), \theta_{w}$ (pitch), $\left.\phi_{w}(\mathrm{yaw})\right)$

- 1 variable from $\mathrm{M}^{\prime}$ to $\mathrm{I}$ in the $x$ direction of $\mathrm{M}^{\prime}$ which is referred as $a$

- 1 variable from $\mathrm{I}$ to $\mathrm{I}^{\prime}$ for rotation around $x$ axis of $\mathrm{I}$, referred by $\alpha$

- 1 variable from $\mathrm{I}^{\prime}$ to $\mathrm{L}^{*}$ in the $z$ direction of $\mathrm{I}$, named $b$

- 1 variable from $\mathrm{L}^{*}$ to $\mathrm{L}^{\prime}$ for rotation around $z$ axis of $\mathrm{L}^{*}$, symbolized by $\beta$

These variables are shown in Fig. 3 and Fig. 4.

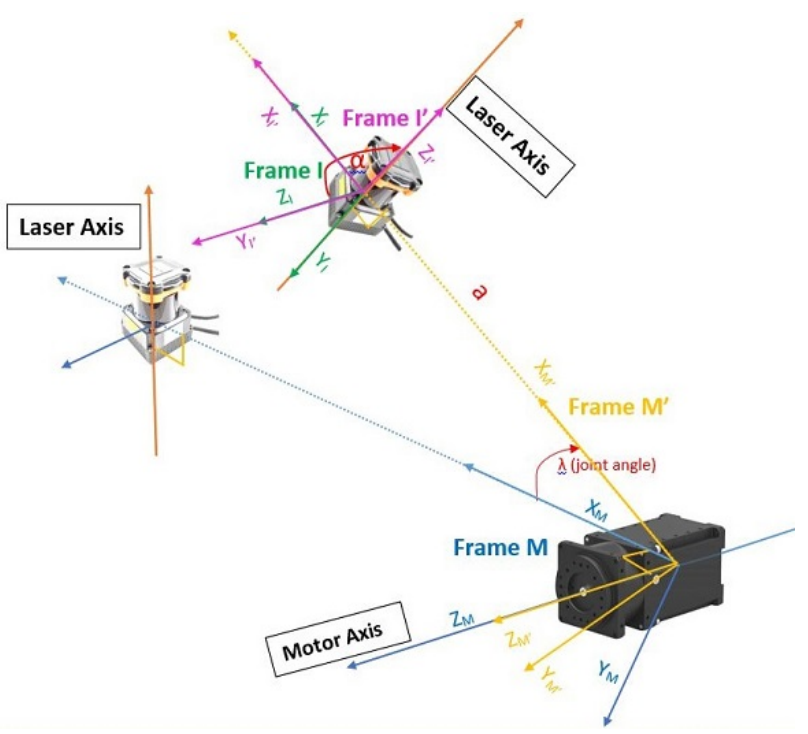

Fig. 3: Coordinate Frames and Variables - Part I

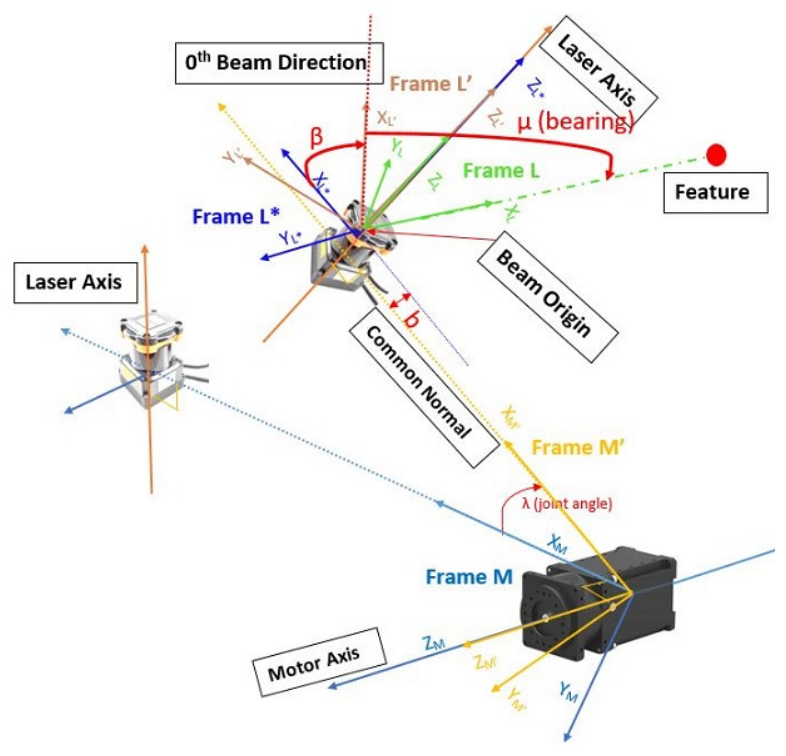

Fig. 4: Coordinate Frames and Variables - Part II

\section{Establishing the World Coordinate Frame (W)}

We artificially populate the environment with a set of reflective markers placed to be visible to the laser but placed at arbitrary locations. Distance between these markers are measured and used to obtain a distance matrix. Multidimensional scaling [22], [23] is then used to establish the locations of these markers with respect to a local coordinate frame. This local coordinate frame is set to W, see Fig. 5 .

The intensity of the laser beam reflected by the markers can be used to identify the feature points and therefore obtain the distance to the markers from the optical centre of the LRF. Sec. IV provides more details on how the marker locations are extracted from the 3D point cloud captured by the sensor.

Let $r_{i}$ be the range measurement to a selected marker located at $\left(x_{i}, y_{i}, z_{i}\right)$ in the world coordinate frame $\mathrm{W}$ 


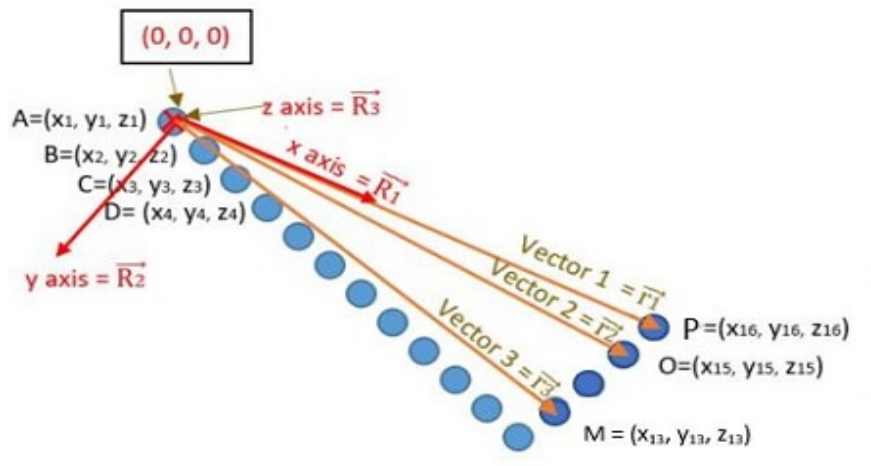

$A=$ Origin (Starting point of the distance matrix)
$P=$ Point with the longest distance from $A$
$O=$ Point with the next longest distance from $A$
after $P$
$x$ axis $=\overrightarrow{R_{1}}=\overrightarrow{r_{1}} /|\overrightarrow{r 1}|$
$z$ axis $=\overrightarrow{R_{3}}=\left(\overrightarrow{r 1} \times \overrightarrow{r_{2}}\right) /\left|\overrightarrow{r 1} \times \overrightarrow{r_{2}}\right|$
$y$ axis $=\overrightarrow{R_{2}}=\left(\overrightarrow{R 3} \times \overrightarrow{R_{1}}\right)$
3D coordinate of point $M$ with respect to the new

3D coordinate of point $M$ with respect to the new
frame established $=\left(\left(\overrightarrow{\mathrm{r}_{3}} \cdot \overrightarrow{\mathrm{R}_{1}}\right),\left(\overrightarrow{\mathrm{r} 3} \cdot \overrightarrow{\mathrm{R}_{2}}\right),\left(\overrightarrow{\mathrm{r}_{3}} \cdot \overrightarrow{\mathrm{R}_{3}}\right)\right)$

Fig. 5: Establishing the arbitrary World Coordinate Frame

as defined in (1). The location of the $i^{t h}$ marker in the coordinate frame $\mathrm{L}$ which is established such that a selected point will always be along its $x$ axis is given by (2).

$$
\begin{aligned}
r_{W} & =\left[\begin{array}{lll}
x_{i} & y_{i} & z_{i}
\end{array}\right] \\
\overrightarrow{r_{L}} & =\left[\begin{array}{lll}
r_{i} & 0 & 0
\end{array}\right]
\end{aligned}
$$

$\overrightarrow{r_{L}}$ can now be transformed to the world coordinate frame W using (3).

$$
\begin{array}{r}
{\left[\begin{array}{rr}
r_{L W} & 1
\end{array}\right]^{\prime}={ }^{W} T_{M} *{ }^{M} T_{M^{\prime}} *{ }^{M^{\prime}} T_{I} *{ }^{I} T_{I^{\prime}} *} \\
{ }^{I^{\prime}} T_{L *} *{ }^{L *} T_{L^{\prime}} *{ }^{L^{\prime}} T_{L} *\left[\begin{array}{ll}
r_{L} & 1
\end{array}\right]^{\prime}
\end{array}
$$

In (3), homogeneous transformation from $\mathrm{W}$ to $\mathrm{M}={ }^{W} T_{M}$, is formed using $\left(x_{w}, y_{w}, z_{w}, \psi_{w}, \theta_{w}, \phi_{w}\right)$.

Homogeneous transform from $\mathrm{M}$ to $\mathbf{M}^{\prime},{ }^{M} T_{M^{\prime}}$ is given by,

$$
{ }^{M} T_{M^{\prime}}=\left(\begin{array}{cccc}
\cos (\lambda) & -\sin (\lambda) & 0 & 0 \\
\sin (\lambda) & \cos (\lambda) & 0 & 0 \\
0 & 0 & 1 & 0 \\
0 & 0 & 0 & 1
\end{array}\right)
$$

where $\lambda$ is the rotation angle corresponding to that position.

Other Homogeneous transforms used in (3) are defined below.

$$
\begin{gathered}
{ }^{\prime} T_{I}=\left(\begin{array}{cccc}
1 & 0 & 0 & a \\
0 & 1 & 0 & 0 \\
0 & 0 & 1 & 0 \\
0 & 0 & 0 & 1
\end{array}\right) \\
{ }^{I} T_{I^{\prime}}=\left(\begin{array}{cccc}
1 & 0 & 0 & 0 \\
0 & \cos (\alpha) & -\sin (\alpha) & 0 \\
0 & \sin (\alpha) & \cos (\alpha) & 0 \\
0 & 0 & 0 & 1
\end{array}\right)
\end{gathered}
$$

$$
\begin{gathered}
{ }^{I^{\prime}} T_{L *}=\left(\begin{array}{cccc}
1 & 0 & 0 & 0 \\
0 & 1 & 0 & 0 \\
0 & 0 & 1 & b \\
0 & 0 & 0 & 1
\end{array}\right) \\
{ }^{L *} T_{L^{\prime}}=\left(\begin{array}{cccc}
\cos (\beta) & -\sin (\beta) & 0 & 0 \\
\sin (\beta) & \cos (\beta) & 0 & 0 \\
0 & 0 & 1 & 0 \\
0 & 0 & 0 & 1
\end{array}\right) \\
{ }^{\prime} T_{L}=\left(\begin{array}{cccc}
\cos (\mu) & -\sin (\mu) & 0 & 0 \\
\sin (\mu) & \cos (\mu) & 0 & 0 \\
0 & 0 & 1 & 0 \\
0 & 0 & 0 & 1
\end{array}\right)
\end{gathered}
$$

where $\mu$ is the bearing angle corresponding to a given point.

\section{Optimization Algorithm}

For a given set of parameters, the Euclidean distance, $d_{d i s}$, between $r_{W}$ and $r_{\overrightarrow{L W}}$ (10) provides a scalar measure of the disparity between these quantities.

$$
d_{d i s}=\left|r_{\overrightarrow{L W}}-r_{W}\right|
$$

The calibration problem is formulated as a non-linear least square optimization problem as shown in (11), and the best estimate for $\left(x_{w}, y_{w}, z_{w}, \psi_{w}, \theta_{w}, \phi_{w}, a, b, \alpha, \beta\right)$ is obtained.

$$
\underset{x_{w}, y_{w}, z_{w}, \psi_{w}, \theta_{w}, \phi_{w}, a, b, \alpha, \beta}{\arg \min } d_{d i s}
$$

In the experiments presented in Sec. IV Matlab implementation of Levenberg-Maquardt algorithm was used to obtain the solution to (11)

\section{EXPERIMENTAL SETUPS}

In order to validate the proposed calibration approach, we conducted experiments in both simulation and real world setups. Robot Operating System (ROS) [24] Kinetic Kame distribution release was used as the middle-ware for collecting experimental data. Experiments on the collected data were conducted in Matlab ${ }^{\circledR}$ R2017a environment. 


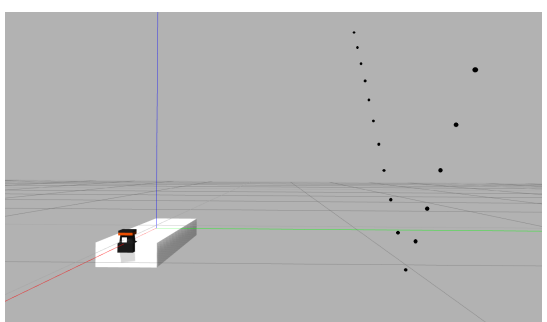

Fig. 6: Simulation set up in Gazebo

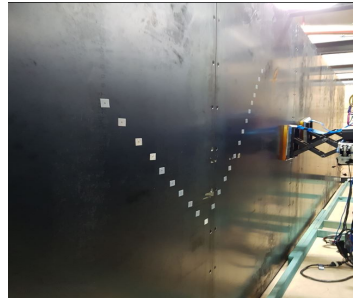

Fig. 7: Feature population inside the UTS test tunnel - V shape

\section{A. Gazebo Simulation}

For simulations, we use the Gazebo robot simulation software with ROS. Spheres of $5 \mathrm{~mm}$ radius with surfaces created from reflective material were populated in the Gazebo environment (Fig. 6) on a planar surface. In order to improve the visual quality of the figure, in Fig. 6, the reflective markers are represented using black circles. A simulated LRF was rotated from $90^{\circ}$ to $-90^{\circ}$ while capturing the $2 \mathrm{D}$ scans of the environment.

\section{B. Real world experiment}

The experiment was conducted in a tunnel structure available at UTS (Fig. 1). An LRF which is rolling around the motor axis is mounted at the rear end of the robot.

The tunnel walls which were planar, were populated with reflective markers in the shape of $\mathrm{V}$ as in Fig. 7. The distances between these reflective points were manually measured. As described in Sec. III-C, the world coordinate frame W was set up (Fig. 5).

Even though the proposed algorithm does not need the reflective markers to be populated in straight lines, we have done so to easily measure the distances.

\section{Processing the laser scans and associating data points}

We use the intensity values associated with each beam point to identify the features. The intensity value of each point in every scan throughout the rotating time period were plotted in a mesh diagram as in Fig. 8. Top view of this mesh plot is shown in Fig. 9. The intensity variation within a $7 \times 7$ grid around a typical reflective feature point is shown in Fig. 10. From this we chose the beam with the maximum intensity to identify the feature. Once the reflective points were selected, scan number, beam number and the associated timestamp values of a particular point were used to correlate it to the corresponding laser scan. The scan number and

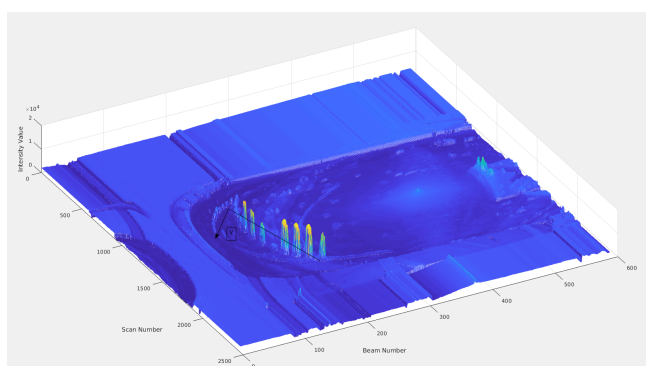

Fig. 8: Mesh Plot of the intensity values

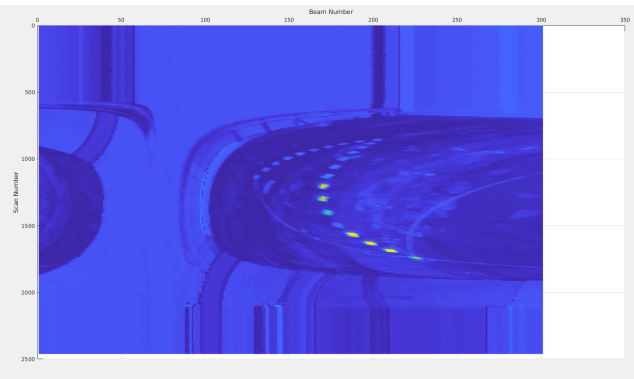

(a) Before Thresholding

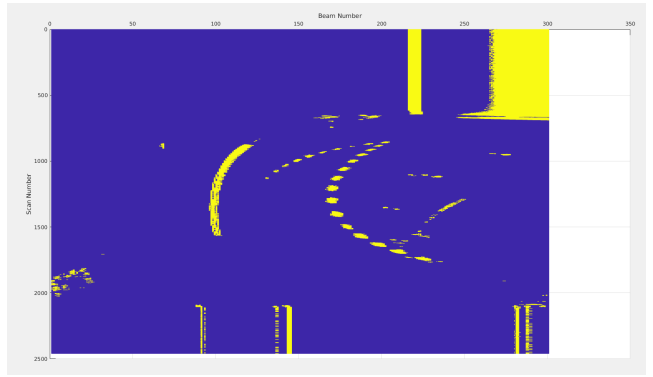

(b) After Thresholding

Fig. 9: Top view of the intensity mesh plot before and after thrsholding

the beam number were used to recover range and bearing measurements while timestamp value was used to get the joint angle of the rotating motor. Since getting an exact timestamp match between recorded laser scan and joint angle values is practically impossible, we use linear interpolation to get the joint angle corresponding to a given timestamp.

Reasonable initial values are assigned to $\left(x_{w}, y_{w}, z_{w}, \psi_{w}, \theta_{w}, \phi_{w}, a, b, \alpha, \beta\right)$ variables to aid the optimiser to reach optimal values efficiently. Parameters $\left(x_{w}, y_{w}, z_{w}, \psi_{w}, \theta_{w}, \phi_{w}\right)$ were estimated by taking rough measurements and approximating the rotation around each

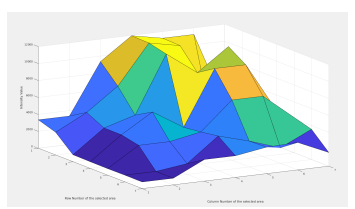

Fig. 10: Surf Plot of the intensity values around a selected point 
TABLE I: Optimiser results of Gazebo simulation: Comparison of DH parameters with the ground truth

\begin{tabular}{|c||c|c|c|}
\hline \hline Variable & Value (0 rotation) & Value (30 rotation) & Ground Truth \\
\hline $\mathrm{a}(\mathrm{mm})$ & -9.0 & -5.8 & -5.0 \\
$\alpha(\mathrm{rad})$ & 1.57 & 1.57 & 1.57 \\
$\mathrm{~b}(\mathrm{~mm})$ & 20.7 & 14.8 & 20.0 \\
$\beta(\mathrm{rad})$ & 1.57 & 1.57 & 1.57 \\
\hline
\end{tabular}

TABLE II: Optimiser results of real laser:

\begin{tabular}{|c||c|}
\hline \hline Variable & $\mathrm{V}$ \\
\hline $\mathrm{a}(\mathrm{mm})$ & 10.09 \\
$\alpha(\mathrm{rad})$ & 1.574 \\
$\mathrm{~b}(\mathrm{~mm})$ & 18.25 \\
$\beta(\mathrm{rad})$ & -1.575 \\
\hline
\end{tabular}

axis of the coordinate frame marked on the tunnel plane. As for the DH parameters, $a$ and $b$ were given initial values which were roughly measured from the set up while $\alpha$ and $\beta$ were assigned with either $+90^{\circ},-90^{\circ}$ or 0 .

\section{RESUltS AND OBSERVATIONS}

Results from Gazebo simulation is shown in Fig. 11. The laser range sensor mount was initially kept at a $0^{\circ}$ rotation and later at a $30^{\circ}$ rotation. Tab. I compares the optimiser generated DH parameters against the Gazebo based ground truths.

The values of $\alpha$ and $\beta$ angles were approximately the same in both setups. However, the $a$ and $b$ values differ. $a$ is decreased nearly by $3 \mathrm{~mm}$ and $b$ by $6 \mathrm{~mm}$. The assumed reason behind this is the size of the spheres which was $5 \mathrm{~mm}$ in radius. When selecting a corresponding point, the selected point is from an entire patch which corresponds to a particular sphere at different beam and joint angles. Hence, the selected point can be one side of the sphere at one location, other side of the sphere at another location and can even be the exact mid point of the sphere. Thus a variation up to $1 \mathrm{~cm}$ can be expected.

Results from real world setup is reported in Fig 12. The DH parameters computed using markers from $\mathrm{V}$ in Tab. II

Using the values of the optimizer results of DH parameters, the collected laser scans were re-projected to create a 3D point cloud of the tunnel shown in Fig. 13. In order to estimate the tunnel dimensions, four major planes (i.e. left wall, right wall, floor and ceiling) were extracted from this re-projected 3D point cloud using RANSAC [25]. The distance from the robot to the manhole plate mounted in front of it is also computed. Tab. III compares these estimated values with average measured values obtained using a laser measuring device.

TABLE III: Estimated UTS test tunnel dimensions using the reprojected point cloud

\begin{tabular}{|c||c|c|}
\hline \hline Variable & Measured & Estimated \\
\hline width $(\mathrm{mm})$ & 780 & 772 \\
height $(\mathrm{m})$ & 2.30 & 2.28 \\
distance to manhole plate $(\mathrm{m})$ & 2.24 & 2.22 \\
\hline
\end{tabular}

\section{CONCLUSION}

This paper presents a solution based on observing a set of arbitrarily placed reflective markers to find the HTM relating the coordinate frame of the robot and the 3D range sensor constructed using 2D LRF whose body is rotated about an axis using a motor. Intensity of the reflected signal from the markers is used to distinguish these from the surrounding. The geometry of the range sensor was represented using DH parameters, providing a minimal set of extrinsic calibration parameters. Accuracy of the initial guess plays a vital role in determining the optimiser output. Nominal values of the DH parameters are typically available in the design drawings of the range sensor set up. Estimating initial guess to the local coordinate frame of the markers was aided by the structure of the environment, which is a tunnel consisting of planes. The accuracy can further be improved if features are populated in a 3D environment. Future work will explore the feasibility of designing a calibration object populated with markers to make it easy to obtain this initial guess as well as to minimise the human errors in measuring distance between markers.

\section{ACKNOWLEDGEMENT}

This work is supported by the NSW Roads and Maritime Services and the Centre for Autonomous Systems (CAS) at the University of Technology Sydney.

We also acknowledge Dr. Buddhi Wijerathna, Dr. ChiaHan Yang and Mr. Matthew Isaac Abbon for their support to conduct experiments at the UTS test rig.

\section{REFERENCES}

[1] "Velodyne LiDar," 2018. [Online]. Available: https://velodynelidar. com

[2] Anthony J. (Intel), "Intel RealSense Technology," 2018. [Online] Available: https://software.intel.com/en-us/realsense

[3] G. Pandey, J. R. McBride, S. Savarese, and R. M. Eustice, "Automatic targetless extrinsic calibration of a $3 \mathrm{~d}$ lidar and camera by maximizing mutual information." in AAAI, 2012.

[4] C. Glennie and D. D. Lichti, "Static calibration and analysis of the velodyne hdl-64e s2 for high accuracy mobile scanning," Remote Sensing, vol. 2, no. 6, pp. 1610-1624, 2010.

[5] P. Fuersattel, C. Plank, A. Maier, and C. Riess, "Accurate laser scanner to camera calibration with application to range sensor evaluation," IPSJ Transactions on Computer Vision and Applications, vol. 9, no. 1, p. 21, 2017.

[6] H. Dong, S. Anderson, and T. D. Barfoot, "Two-axis scanning lidar geometric calibration using intensity imagery and distortion mapping," in Robotics and Automation (ICRA), 2013 IEEE International Conference on. IEEE, 2013, pp. 3672-3678.

[7] V. Pradeep, K. Konolige, and E. Berger, "Calibrating a multi-arm multi-sensor robot: A bundle adjustment approach," in Experimental robotics. Springer, 2014, pp. 211-225.

[8] "Work Health and Safety Regulation 2017," 2017. [Online]. Available: https://www.legislation.nsw.gov.au/\#/view/regulation/2017/404

[9] J. Morales, J. L. Martínez, A. Mandow, A. J. Reina, A. PequeñoBoter, and A. García-Cerezo, "Boresight calibration of construction misalignments for 3D scanners built with a 2D laser rangefinder rotating on its optical center," Sensors (Switzerland), 2014.

[10] D. W. Kim, H. S. Kim, and J. E. Ha, "Extrinsic calibration of rotating laser range finder using scene structure," in 2013 13th International Conference on Control, Automation and Systems (ICCAS 2013), Oct 2013, pp. 701-703.

[11] E. Krotkov, "Laser rangefinder calibration for a walking robot," in Proceedings. 1991 IEEE International Conference on Robotics and Automation, Apr 1991, pp. 2568-2573 vol.3. 

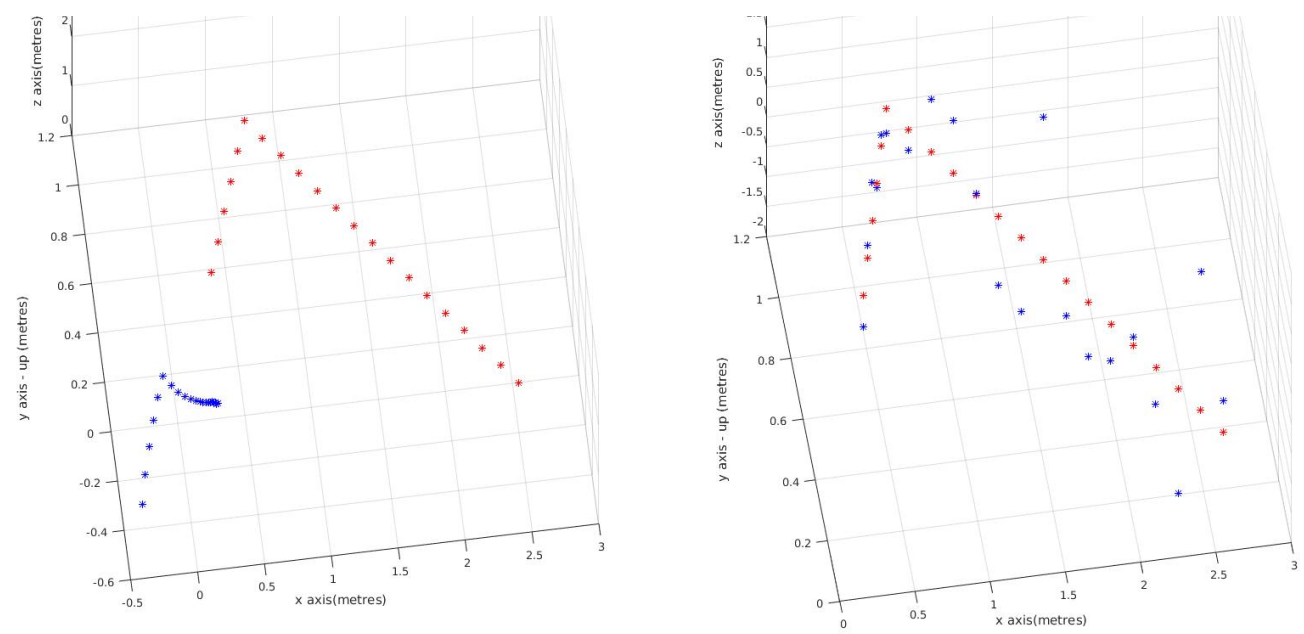

Fig. 11: Optimization results from Gazebo simulation set up, Before (left) and After Optimization (right)

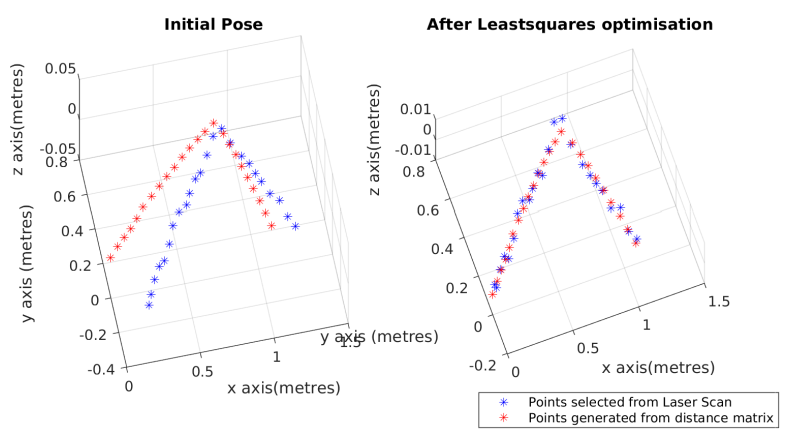

Fig. 12: Optimization results from real world experiments at UTS test tunnel

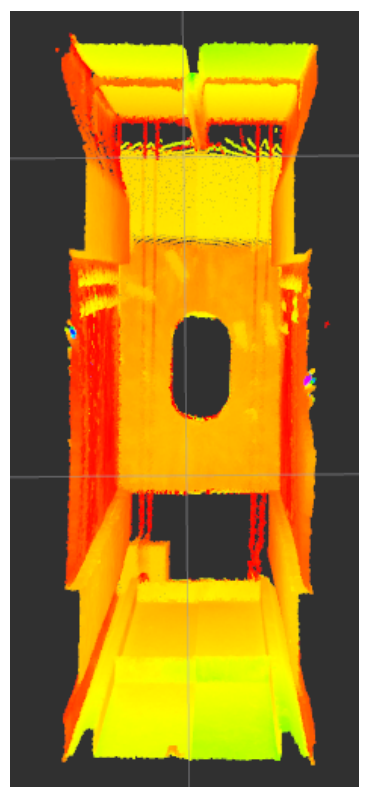

Fig. 13: Reprojected 3D point cloud of the tunnel
[12] S. Wasielewski and O. Strauss, "Calibration of a multi-sensor system laser rangefinder/camera," in Intelligent Vehicles '95 Symposium., Proceedings of the, Sep 1995, pp. 472-477.

[13] L. Yu, M. Peng, Z. You, Z. Guo, P. Tan, and K. Zhou, "Separated calibration of a camera and a laser rangefinder for robotic heterogeneous sensors," International Journal of Advanced Robotic Systems, 2013.

[14] Q. Zhang and R. Pless, "Extrinsic calibration of a camera and laser range finder (improves camera calibration)," in 2004 IEEE/RSJ International Conference on Intelligent Robots and Systems (IROS) (IEEE Cat. No.04CH37566), vol. 3, Sept 2004, pp. 2301-2306 vol.3.

[15] R. Unnikrishnan and M. Hebert, "Fast Extrinsic Calibration of a Laser Rangefinder to a Camera," Robotics Institute, 1 2005. [Online]. Available: http://repository.cmu.edu/robotics/339

[16] E. W. Y. So, F. Basso, and E. Menegatti, "Calibration of a rorating 20 laser range-finder using point-plane coistraints," Journal of Automation Mobile Robotics and Intelligent Systems, vol. 7, no. 2, pp. 30-38, 2013.

[17] L. Kurnianggoro, V.-D. Hoang, and K.-H. Jo, "Calibration of a 2 d laser scanner system and rotating platform using a point-plane constraint," Computer Science and Information Systems, vol. 12, no. 1, pp. 307$322,2015$.

[18] J. Kang and N. L. Doh, "Full-dof calibration of a rotating 2-d lidar with a simple plane measurement," IEEE Transactions on Robotics, vol. 32 , no. 5, pp. 1245-1263, 2016.

[19] M. M. Bykov and V. S. Tyurin, "Increase of measurement accuracy of distances by laser rangefinder with adaptive threshold," in Proceedings of CAOL 2005. Second International Conference on Advanced Optoelectronics and Lasers, 2005., vol. 2, Sept 2005, pp. 297-299 vol. 2.

[20] M. Sheehan, A. Harrison, and P. Newman, "Self-calibration for a 3D laser," The International Journal of Robotics Research, 2012

[21] C. Glennie and D. D. Lichti, "Static calibration and analysis of the velodyne HDL-64E S2 for high accuracy mobile scanning," Remote Sensing, 2010.

[22] J. C. Gower, "Some distance properties of latent root and vector methods used in multivariate analysis," Biometrika, vol. 53, no. 3-4, pp. 325-338, 1966.

[23] T. F. Cox and M. A. Cox, Multidimensional scaling. CRC press, 2000

[24] M. Quigley, K. Conley, B. P. Gerkey, J. Faust, T. Foote, J. Leibs, R. Wheeler, and A. Y. $\mathrm{Ng}$, "Ros: an open-source robot operating system," in ICRA Workshop on Open Source Software, 2009.

[25] M. A. Fischler and R. C. Bolles, "Random sample consensus: a paradigm for model fitting with applications to image analysis and automated cartography," in Readings in computer vision. Elsevier, 1987, pp. 726-740. 